\title{
Electric and magnetic wakefields in a plasma channel
}

\author{
Pallavi Jha, Punit Kumar, Ajay K. Upadhyaya, and Gaurav Raj \\ Department of Physics, University of Lucknow, Lucknow-226007, India
}

(Received 29 November 2004; published 18 July 2005)

\begin{abstract}
A detailed analytical study of plasma wakefield generation in a wide parabolic plasma channel is reported. A perturbative technique involving orders of the incident laser beam and the effects of inhomogeneity of the plasma density is used to obtain explicit electric as well as magnetic wakefields. The axial and transverse forces acting on a test electron due to the wakefields have been evaluated.
\end{abstract}

DOI: $10.1103 /$ PhysRevSTAB.8.071301

PACS numbers: $52.38 . \mathrm{Kd}$

\section{INTRODUCTION}

Interaction of intense laser pulses with plasma has the capability of setting up enormous acceleration gradients $(\approx \mathrm{GV} / \mathrm{m})$ in which electrons and ions can be accelerated to very high energies. The pioneering work of Tajima and Dawson [1] has given a great impetus to the idea of electron acceleration using intense laser beams. In a laser wakefield accelerator (LWFA) [2-7] a high intensity laser pulse with a duration $\left(\tau_{L}\right)$ approximately equal to a plasma oscillation period $\left(2 \pi / \omega_{p}\right)$ generates a large amplitude plasma wave with a phase velocity that is close to the speed of light. A beam of electrons interacting with this wave can be effectively accelerated. Recently, direct observation of wakefields [8] and acceleration of injected electrons [9] in LWFA configuration have been reported.

Extended propagation of the laser pulse is necessary to achieve high electron energy. In the absence of optical guiding the acceleration distance is limited to a few Rayleigh lengths which is far below that necessary to reach $\mathrm{GeV}$ electron energies $[2,10]$. In a preformed plasma channel the laser pulse can propagate upto several Rayleigh lengths without substantial spreading [11-13]. Therefore, generation of wakefields in a preformed channel becomes a subject of great interest [14-16].

Earlier workers have reported simulation studies on wakefields generated in wide parabolic plasma channels $[17,18]$. The present paper is devoted to a detailed analytical study of plasma wakefield generation in a parabolic plasma channel. We use a perturbative technique involving orders of the incident laser beam and the effects of inhomogeneity of the plasma density to obtain explicit electric as well as magnetic wakefields. The results distinctly point out the effect of the channel (being used for purposes of focusing) on the generated wakefields. Such a study has not been reported in literature before.

In Sec. II the lowest order fast oscillating plasma electron velocities and density perturbations have been derived. Time averaged current densities are coupled with Maxwell's equations in Sec. III, leading to the generation of electric and magnetic wakefields. Here, the quasistatic approximation (QSA) [11] has been used. In Sec. IV the axial and radial forces acting on a test electron moving along the $z$ direction are derived and the effect of the channel is discussed. Section V presents summary and discussion.

\section{HIGH FREQUENCY PLASMA OSCILLATIONS}

Consider a linearly polarized laser beam, represented by the electric vector $\vec{E}=\hat{e}_{x} E(r, z, t) \cos \left(k_{o} z-\omega_{o} t\right)\left(\hat{e}_{x}\right.$ is the unit vector of polarization), propagating in a plasma channel having density profile $n^{(0)}\left(=n^{(0,0)}+n^{(0,1)}=\right.$ $\left.n_{o}+\Delta n r^{2} / r_{\mathrm{ch}}^{2}\right)$. Here $n_{o}\left(=n^{(0,0)}\right)$ is the ambient plasma density, $\Delta n r^{2} / r_{\text {ch }}^{2}\left(=n^{(0,1)}\right)$ represents radial variation in channel density, $\Delta n$ is the channel depth, and $r_{\text {ch }}$ is the channel radius. Using perturbative technique orders of various parameters are considered such that the first index of the superscript gives the order of the field amplitude whereas the second index arises due to the presence of the channel. The condition for validity of perturbative expansions due to the presence of the channel is $k_{\mathrm{po}} r_{\mathrm{ch}}>1$. The plasma electrons obey the equations

$$
\frac{\partial \vec{v}}{\partial t}=-\frac{e}{m} \vec{E}-\frac{e}{m}\left(\frac{\vec{v}}{c} \times \vec{B}\right)-\frac{1}{2} \vec{\nabla}(\vec{v} \cdot \vec{v})+\vec{v} \times(\vec{\nabla} \times \vec{v}),
$$

$$
\frac{\partial n}{\partial t}+\vec{\nabla} \cdot(n \vec{v})=0
$$

and

$$
\vec{\nabla} \cdot \vec{E}=-4 \pi e\left(n-n^{(0)}\right) .
$$

Equations (1)-(3) are perturbatively expanded for the first order of the electromagnetic field. Further perturbations due to the presence of the channel (represented by the second index) lead to

$$
\begin{aligned}
\frac{\partial v^{(1,0)}}{\partial t} & =-\frac{e}{m} E \cos \left(k_{o} z-\omega_{o} t\right) ; \\
\boldsymbol{v}^{(1,0)} & =c a \sin \left(k_{o} z-\omega_{o} t\right) \\
\frac{\partial n^{(1,0)}}{\partial t}+\vec{\nabla} \cdot n^{(0,0)} \overrightarrow{\boldsymbol{v}}^{(1,0)} & =0 ; \\
n^{(1,0)} & =0,
\end{aligned}
$$


[where $a=e E / m c \omega_{o}$ and transverse Coulomb gauge $(\vec{\nabla}$. $\vec{E}=0$ ) has been used] and

$$
\begin{aligned}
\frac{\partial v^{(1,1)}}{\partial t} & =-\frac{e}{m} \vec{E}^{(1,1)}, \\
\frac{\partial n^{(1,1)}}{\partial t}+\vec{\nabla} \cdot\left(n^{(0,0)} \overrightarrow{\boldsymbol{v}}^{(1,1)}+n^{(0,1)} \overrightarrow{\boldsymbol{v}}^{(1.0)}\right) & =0, \\
\vec{\nabla} \cdot \vec{E}^{(1,1)} & =-4 \pi e n^{(1,1)} .
\end{aligned}
$$

Differentiating Eq. (5b) with respect to time and substituting Eqs. (5a) and (5c) gives

$$
\begin{aligned}
\frac{\partial^{2} n^{(1,1)}}{\partial t^{2}}+\omega_{\mathrm{po}}^{2} n^{(1,1)} & =\frac{e}{m}\left(\vec{\nabla} n^{(0,1)}\right) \cdot \vec{E}^{(1,0)} \\
& =\frac{e}{m} \frac{2 \Delta n x E}{r_{\mathrm{ch}}^{2}} \cos \left(k_{o} z-\omega_{o} t\right)
\end{aligned}
$$

where $\omega_{\mathrm{po}}^{2}=4 \pi n_{o} e^{2} / m$. Equation (6) represents forced oscillations of the perturbed plasma density driven by a force having frequency $\omega_{o}$ and amplitude $2 \Delta n x e E / r_{\mathrm{ch}}^{2} m$ and its solution is given by

$$
n^{(1,1)}=-R x a \cos \left(k_{o} z-\omega_{o} t\right)
$$

where $R=2 \Delta n c \omega_{o} / r_{\mathrm{ch}}^{2}\left(\omega_{o}^{2}-\omega_{\mathrm{po}}^{2}\right)$. Thus first order perturbations are introduced in the plasma density due to the presence of the channel. These oscillations arise due to the interaction of the electron quiver velocity with the gradient of the channel density profile. Thus, $\vec{E} \cdot \vec{\nabla} n^{(0,1)} \neq 0$ provides a periodic force and causes the plasma electrons to oscillate with the frequency of laser. This high frequency fluctuation in charge density leads to generation of a longitudinal electric field and velocity, respectively, given by

$$
E_{h z}^{(1,1)}=\frac{m}{e} P \omega_{o} x a \sin \left(k_{o} z-\omega_{o} t\right)
$$

and

$$
\boldsymbol{v}_{z}^{(1,1)}=-\operatorname{Pax} \cos \left(k_{o} z-\omega_{o} t\right),
$$

where $P=\left[\omega_{\mathrm{po}}^{2} /\left(\omega_{o}^{2}-\omega_{\mathrm{po}}^{2}\right)^{3 / 2}\right]\left(2 \Delta n c^{2} / n_{o} r_{\mathrm{ch}}^{2}\right)$.

\section{WAKEFIELD GENERATION}

In order to study the excitation of plasma waves we use the quasistatic approximation. In this approximation, the plasma fluid equations are written in terms of independent variables $\xi=z-c t$ and $\tau=t$. Further it is assumed that the laser does not evolve significantly as it transits a plasma electron [11]. Thus plasma electrons experience a laser field which is a function of $\xi$ and $r$ variables only. Electric and magnetic wakefields can be obtained using the time dependent Maxwell's equations

$$
\begin{aligned}
& \vec{\nabla} \times \vec{E}=-\frac{1}{c} \frac{\partial \vec{B}}{\partial t}, \\
& \vec{\nabla} \times \vec{B}=\frac{4 \pi}{c} \vec{J}+\frac{1}{c} \frac{\partial \vec{E}}{\partial t} .
\end{aligned}
$$

The laser field has a radial Gaussian profile and the generated fields are assumed to be axisymmetric. Thus the nonzero field components may be written as

$$
\begin{aligned}
\frac{\partial E_{r}}{\partial \xi}-\frac{\partial E_{z}}{\partial r} & =\frac{\partial B_{\theta}}{\partial \xi}, \\
\frac{\partial E_{r}}{\partial \xi}-\frac{\partial B_{\theta}}{\partial \xi} & =\frac{4 \pi}{c} J_{r}, \\
\frac{\partial E_{z}}{\partial \xi} & =\frac{4 \pi}{c} J_{z}-\frac{1}{r} \frac{\partial\left(r B_{\theta}\right)}{\partial r},
\end{aligned}
$$

where $J_{r}$ and $J_{z}$ are transverse and axial current densities, respectively. The second order slow components of the plasma electron velocity are obtained by substituting the first order quantities into the Lorentz force equation as follows,

$$
\frac{\partial v_{z}}{\partial \xi}=\frac{e}{m c} E_{z}+\frac{1}{4} \frac{\partial}{\partial \xi}\left(c a^{2}+\frac{P^{2} r^{2} a^{2}}{2 c}\right)
$$

and

$$
\frac{\partial v_{r}}{\partial \xi}=\frac{e}{m c} E_{r}-\frac{r}{r_{o}^{2}} c a^{2} .
$$

$E_{z}$ and $E_{r}$ represent the axial and radial electric wakefields. The last term on the right-hand side of Eq. (10) is obtained by substituting the value of $\boldsymbol{v}_{z}^{(1,1) 2}$ averaged over the polar angle $\theta$. In deriving Eqs. (10) and (11) it is found that the ponderomotive nonlinear effects $[\vec{\nabla}(\vec{v} \cdot \vec{v})]$ contribute to transverse as well as longitudinal wakefield generation. However, nonlinear terms $\vec{v} \times \vec{B}$ and $\vec{v} \times(\vec{\nabla} \times \vec{v})$ representing vortex motion contribute only to transverse velocity components. Further, the ponderomotive force is modified due to the contribution of first order longitudinal velocity of plasma electrons. Combining Eqs. (9c) and (10) gives

$$
\begin{aligned}
{\left[\frac{\partial^{2}}{\partial \xi^{2}}+k_{p}^{2}\right] E_{z}=} & -\frac{m c^{2} k_{p}^{2}}{4 e}\left(1+\frac{P^{2} r^{2}}{2 c^{2}}+\frac{P R r^{2}}{n^{(0)} c}\right) \frac{\partial a^{2}}{\partial \xi} \\
& -\frac{\partial}{\partial \xi}\left(\frac{1}{r} \frac{\partial}{\partial r} r B_{\theta}\right),
\end{aligned}
$$

where $k_{p}^{2}=k_{\mathrm{po}}^{2}\left[1+\left(\Delta n r^{2} / n_{o} r_{\mathrm{ch}}^{2}\right)\right]$. Also coupling Eqs. (9a) and (9b) and substituting Eq. (11) gives

$$
E_{r}=-\frac{1}{k_{p}^{2}} \frac{\partial^{2} E_{z}}{\partial \xi \partial r}+\frac{m r c^{2} a^{2}}{e r_{o}^{2}} .
$$

Equations (12) and (13) are the governing equations for generation of axial and transverse electric wakefields in a plasma channel. The magnetic wakefields can be subse- 
quently obtained from Eq. (9a). It may be noted that these fields are of the second order of the radiation field. Therefore, in the analysis that follows the first superscript pertaining to the order of the electromagnetic field is dropped for the sake of convenience.

For a wide channel $k_{\mathrm{po}} r_{\mathrm{ch}}>1$, the generated wakefields can be perturbatively expanded in orders of inhomogeneity of channel. Also the radial dependence of transverse electric and magnetic wakefields can be considered to be a higher order effect. Thus the lowest order equation for axial wakefield is given by

$$
\left(\frac{\partial^{2}}{\partial \xi^{2}}+k_{\mathrm{po}}^{2}\right) E_{z}^{(0)}=-\frac{m c^{2} k_{\mathrm{po}}^{2}}{4 e} \frac{\partial a^{2}}{\partial \xi}
$$

For a pulse profile of the form $a^{2}=a_{r}^{2} \sin ^{2} \pi \xi / L$, where $a_{r}^{2}=a_{o}^{2} \exp \left(-2 r^{2} / r_{o}^{2}\right)$, the solutions to Eq. (14) within the laser pulse $(0 \leq \xi \leq L)$ and behind the pulse $(\xi<0)$ are, respectively, given by

$$
\begin{aligned}
& E_{z}^{(0)}=\frac{\varepsilon k_{\mathrm{po}} f}{8}\left[\sin k_{\mathrm{po}}(L-\xi)+\frac{k_{\mathrm{po}} L}{2 \pi} \sin \frac{2 \pi \xi}{L}\right] \\
& E_{z}^{(0)}=\frac{\varepsilon k_{\mathrm{po}} f}{8}\left[\sin k_{\mathrm{po}}(L-\xi)+\sin k_{\mathrm{po}} \xi\right],
\end{aligned}
$$

where $\varepsilon=m c^{2} a_{r}^{2} / e$ and $f=\left(1-k_{\mathrm{po}}^{2} L^{2} / 4 \pi^{2}\right)^{-1}$. These fields are the same as the axial wakefields generated in homogeneous plasma [19]. From Eq. (13) the lowest order transverse electric wakefields within and behind the pulse are given by

$$
\begin{aligned}
E_{r}^{(0)}= & -\frac{r \varepsilon}{2 r_{o}^{2}}\left[f \cos k_{\mathrm{po}}(L-\xi)+(1-f) \cos \left(\frac{2 \pi \xi}{L}\right)-1\right] \\
& \text { and } \\
E_{r}^{(0)}= & -\frac{r \varepsilon}{2 r_{o}^{2}}\left[f \cos k_{\mathrm{po}}(L-\xi)+\cos \left(\frac{2 \pi \xi}{L}\right)\right. \\
& \left.-f \cos k_{\mathrm{po}} \xi-1\right]
\end{aligned}
$$

respectively. Substituting Eqs. (15) and (16) into Eq. (9a) the lowest order magnetic wakefield, within the pulse, $B_{\theta}^{(0)}=0$, whereas behind the pulse the field is given by

$$
B_{\theta}^{(0)}=-\frac{\varepsilon r}{2 r_{o}^{2}}\left(\cos \frac{2 \pi \xi}{L}-1\right)
$$

This field is zero on axis $(r=0)$.

The first order equation for the axial wakefield is obtained from Eq. (12) as

$$
\begin{aligned}
\left(\frac{\partial^{2}}{\partial \xi^{2}}+k_{\mathrm{po}}^{2}\right) E_{z}^{(1)}= & -k_{\mathrm{po}}^{2} \frac{\Delta n r^{2}}{n_{o} r_{\mathrm{ch}}^{2}} E_{z}^{(0)}-\frac{\Delta n m c^{2} k_{\mathrm{po}}^{2} a_{r}^{2} r^{2} \pi}{4 e n_{o} r_{\mathrm{ch}}^{2} L} \\
& \times \sin \frac{2 \pi \xi}{L}-\frac{\partial}{\partial \xi}\left(\frac{1}{r} \frac{\partial\left(r B_{\theta}^{(0)}\right)}{\partial r}\right)
\end{aligned}
$$

Substitution of the zeroth order quantities leads to first order longitudinal fields within and at the back of the pulse, respectively, as

$$
\begin{aligned}
E_{z}^{(1)}= & \frac{\varepsilon g r^{2} f k_{\mathrm{po}}}{8}\left[\frac{k_{\mathrm{po}}}{2}(L-\xi) \cos k_{\mathrm{po}}(L-\xi)+\left(f-\frac{1}{2}\right) \sin k_{\mathrm{po}}(L-\xi)+\frac{f k_{\mathrm{po}} L}{2 \pi} \sin \frac{2 \pi \xi}{L}\right] \text { and } \\
E_{z}^{(1)}= & -\varepsilon f\left\{\frac{k_{\mathrm{po}} g r^{2}}{16} \sin k_{\mathrm{po}} L \cos k_{\mathrm{po}} \xi-\frac{k_{\mathrm{po}}^{2} g r^{2} L}{16} \cos k_{\mathrm{po}}(L-\xi)-\frac{k_{\mathrm{po}} g r^{2}}{32} \sin k_{\mathrm{po}}(2 L-\xi)+\frac{k_{\mathrm{po}}^{2} g r^{2} L}{16} \cos k_{\mathrm{po}} \xi\right. \\
& \left.-\left[\frac{5 k_{\mathrm{po}} g r^{2}}{32}+\frac{1}{k_{\mathrm{po}} r_{o}^{2}}\left(1-\frac{2 r^{2}}{r_{o}^{2}}\right)\right] \sin k_{\mathrm{po}} \xi-\left[\frac{k_{\mathrm{po}} g r^{2}}{8}+\frac{1}{k_{\mathrm{po}} r_{o}^{2}}\left(1-\frac{2 r^{2}}{r_{o}^{2}}\right)\right] \sin k_{\mathrm{po}}(L-\xi)\right\}
\end{aligned}
$$

where $g=\left(\Delta n / n_{o} r_{\mathrm{ch}}^{2}\right)$. Equation (18a) gives the modification of the longitudinal wakefield due to the presence of the plasma channel within the laser pulse. It may be noted that the perturbed axial wakefield inside the pulse is zero on axis $(r=0)$. However the perturbed longitudinal wakefield behind the laser pulse has contributions due to the presence of the channel as well as the gradient of the magnetic field given by Eq. (16c). Therefore the on-axis field [as given by Eq. (18b)] is nonzero.

The first order transverse electric wakefields within and at the back of the pulse are obtained by substituting Eq. (18) into Eq. (13), as 


$$
\begin{aligned}
E_{r}^{(1)}= & \frac{\varepsilon g f}{2}\left[\left\{\frac{r^{3}}{r_{o}^{2}}+\frac{f r}{2}\left(1-\frac{2 r^{2}}{r_{o}^{2}}\right)\right\} \cos k_{\mathrm{po}}(L-\xi)-\frac{k_{\mathrm{po}} r}{4}\left(1-\frac{2 r^{2}}{r_{o}^{2}}\right)(L-\xi) \sin k_{\mathrm{po}}(L-\xi)\right. \\
& \left.-\left\{\frac{r^{3}}{r_{o}^{2}}+\frac{f r}{2}\left(1-\frac{2 r^{2}}{r_{o}^{2}}\right)\right\} \cos \frac{2 \pi \xi}{L}\right] \text { and } \\
E_{r}^{(1)}= & \varepsilon f\left[-\frac{g r}{8}\left(1-\frac{2 r^{2}}{r_{o}^{2}}\right) \sin k_{\mathrm{po}} L \cdot \sin k_{\mathrm{po}} \xi-\frac{g L k_{\mathrm{po}} r}{8}\left(1-\frac{2 r^{2}}{r_{o}^{2}}\right) \sin k_{\mathrm{po}}(L-\xi)+\frac{g r}{16}\left(1-\frac{2 r^{2}}{r_{o}^{2}}\right) \cos k_{\mathrm{po}}(2 L-\xi)\right. \\
& -\left\{\frac{g r}{16}\left(1-\frac{2 r^{2}}{r_{o}^{2}}\right)+\frac{g r}{4}-\frac{8 r}{k_{\mathrm{po}}^{2} r_{o}^{4}}\left(1-\frac{r^{2}}{r_{o}^{2}}\right)\right\} \cos k_{\mathrm{po}} \xi-\frac{2 r}{8}\left(1-\frac{2 r^{2}}{r_{o}^{2}}\right) k_{\mathrm{po}} L \sin k_{\mathrm{po}} \xi \\
& \left.+\left\{\frac{g r}{4}-\frac{8 r}{k_{\mathrm{po}}^{2} r_{o}^{4}}\left(1-\frac{r^{2}}{r_{o}^{2}}\right)\right\} \cos k_{\mathrm{po}}(L-\xi)\right]
\end{aligned}
$$

respectively. Also, substituting Eqs. (18) and (19) into Eq. (9a) give the first order magnetic wakefields within and behind the pulse:

$$
\begin{aligned}
& B_{\theta}^{(1)}=\frac{\varepsilon g r f}{4}\left[\cos k_{\mathrm{po}}(L-\xi)-\cos \frac{2 \pi \xi}{L}-\cos k_{\mathrm{po}} L+1\right] \quad \text { and } \\
& B_{\theta}^{(1)}=\frac{\varepsilon g r^{3} f}{2 r_{o}^{2}}\left[\cos k_{\mathrm{po}}(L-\xi)-\cos k_{\mathrm{po}} \xi-\cos k_{\mathrm{po}} L+1\right] .
\end{aligned}
$$

Second and higher order wakefields may be obtained similarly.

\section{ACCELERATION AND FOCUSING OF A TEST ELECTRON}

The axial wakefield is maximum when the laser pulse length is nearly equal to the plasma wavelength $L \approx \lambda_{p}$. Using Eqs. (15) and (18) the axial wakefields within and behind the laser pulse in the limit $L \rightarrow \lambda_{p}$ are given by

$$
\begin{aligned}
& E_{z m}=E_{z m}^{(0)}+E_{z m}^{(1)}=-\frac{\varepsilon \pi^{2}}{4 L}\left[\left(1-\frac{\xi}{L}\right) \cos \frac{2 \pi \xi}{L}+\frac{1}{2 \pi} \sin \frac{2 \pi \xi}{L}+g r^{2}\left\{\frac{1}{4}\left(1-\frac{\xi}{L}\right) \cos \frac{2 \pi \xi}{L}\right.\right. \\
& \left.\left.+\left[\frac{\pi}{2}\left(1-\frac{\xi}{L}\right)^{2}+\frac{1}{8 \pi}\right] \sin \frac{2 \pi \xi}{L}\right\}\right] \quad(0 \leq \xi \leq L) \\
& =-\frac{\varepsilon \pi^{2}}{4 L} \cos \frac{2 \pi \xi}{L}-\varepsilon\left[\frac{g r^{2} \pi^{3}}{4 L} \sin \frac{2 \pi \xi}{L}+\left\{\frac{g r^{2} \pi^{2}}{4 L}+\frac{L}{2 r_{o}^{2}}\left(1-\frac{2 r^{2}}{r_{o}^{2}}\right)\right\} \cos \frac{2 \pi \xi}{L}\right] . \quad(\xi<0)
\end{aligned}
$$

In order to study the acceleration mechanism of the LWFA, we consider a test electron moving along the $z$ direction of the laser plasma interaction region. Because of axial wakefields the maximum force experienced by the electron is given by

$$
F_{z}=-e E_{z m}
$$

The electron will be accelerated by this force if $F_{z}>0$. The evolution of the normalized force $\left(F_{z} / m c \omega_{\mathrm{po}}\right)$ with $\xi / L$ (for a matched laser pulse $\Delta n=r_{\mathrm{ch}}^{2} m c^{2} / \pi r_{o}^{4} e^{2}$ ) is shown in Fig. 1 for $a_{o}^{2}=0.1, \lambda_{p}=15.0 \mu \mathrm{m}, r_{o}=$ $15.0 \mu \mathrm{m}$, and $r=4.0 \mu \mathrm{m}$. It is seen that the longitudinal plasma wave exerts an oscillating force on the electron. The solid and dashed curves represent the force acting on the electron in a plasma channel and homogeneous plasma, respectively. The peak force experienced by the electron due to wakefield generated behind the pulse is larger in a plasma channel in comparison to homogeneous plasma by about $19 \%$. However, a test electron moving with the pulse experiences nearly equal forces in homogeneous plasma as well as in a plasma channel, due to the wakefield generated within the pulse. Normalized axial force $\left(F_{z} / m c \omega_{\mathrm{po}}\right)$ acting on a test electron behind the matched laser pulse for a given value of $\xi / L(=-1.0)$ is plotted against $r$ (in $\mu \mathrm{m}$ ) in Fig. 2 (for the same laser beam and plasma parameters as in Fig. 1). It is seen that axial force due to longitudinal wakefield is greater in plasma channel as compared to homogeneous plasma. However as the distance from the axis of the channel increases, the difference in the two forces reduces.

The maximum transverse electric wakefield for $L \rightarrow \lambda_{p}$ is given by 


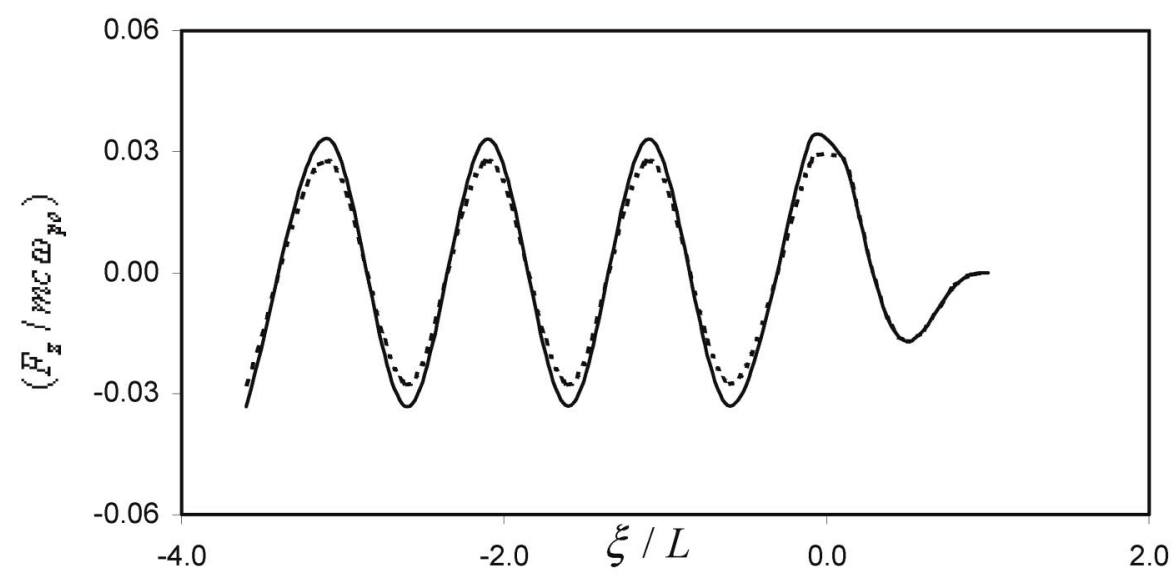

FIG. 1. Normalized axial force $\left(F_{z} / m c \omega_{\mathrm{po}}\right)$ generated by a matched laser pulse, as a function of $\xi / L$ in plasma channel (solid curve) and in a homogeneous plasma (dashed curve) for $a_{o}^{2}=0.1, \lambda_{p}=15.0 \mu \mathrm{m}, r_{o}=15.0 \mu \mathrm{m}$, and $r=4.0 \mu \mathrm{m}$.

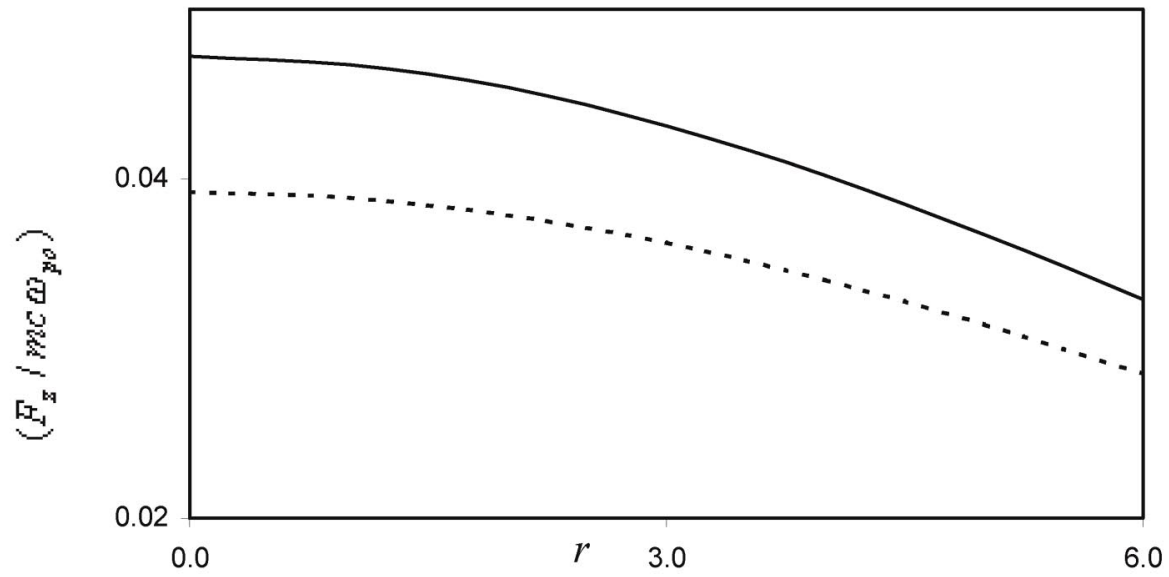

FIG. 2. Normalized axial force $\left(F_{z} / m c \omega_{\mathrm{po}}\right)$ generated by a matched laser pulse, as a function of $r$ (in $\mu \mathrm{m}$ ) in plasma channel (solid curve) and in a homogeneous plasma (dashed curve) for $a_{o}^{2}=0.1, \lambda_{p}=15.0 \mu \mathrm{m}, r_{o}=15.0 \mu \mathrm{m}$, and $\xi / L=-1.0$.

$$
\begin{aligned}
E_{r m}=E_{r m}^{(0)}+E_{r m}^{(1)}= & \frac{\varepsilon r}{2 r_{o}^{2}}\left[\pi\left(1-\frac{\xi}{L}\right) \sin \frac{2 \pi \xi}{L}-\cos \frac{2 \pi \xi}{L}+1\right]-\frac{\varepsilon g \pi}{2}\left[\left\{\frac{r^{3}}{r_{o}^{2}}+\frac{3 r}{8}\left(1-\frac{2 r^{2}}{r_{o}^{2}}\right)\right\}\left(1-\frac{\xi}{L}\right) \sin \frac{2 \pi \xi}{L}\right. \\
& \left.-\frac{\pi r}{4}\left(1-\frac{2 r^{2}}{r_{o}^{2}}\right)\left(1-\frac{\xi}{L}\right)^{2} \cos \frac{2 \pi \xi}{L}\right], \quad(0 \leq \xi \leq L) \\
= & \frac{\varepsilon r}{2 r_{o}^{2}}\left[\pi \sin \frac{2 \pi \xi}{L}-\cos \frac{2 \pi \xi}{L}+1\right]+\frac{\varepsilon \pi}{2}\left[\left\{-\frac{g r}{2}+\frac{4 L^{2} r}{\pi^{2} r_{o}^{4}}\left(1-\frac{r^{2}}{r_{o}^{2}}\right)\right\} \sin \frac{2 \pi \xi}{L}\right. \\
& \left.+\frac{\pi g r}{2}\left(1-\frac{2 r^{2}}{r_{o}^{2}}\right) \cos \frac{2 \pi \xi}{L}\right]
\end{aligned}
$$

and magnetic wakefield is given by

$$
\begin{aligned}
B_{\theta m} & =B_{\theta m}^{(0)}+B_{\theta m}^{(1)}=-\frac{\varepsilon g r \pi}{4}\left(1-\frac{\xi}{L}\right) \sin \frac{2 \pi \xi}{L}, \quad(0 \leq \xi \leq L) \\
& =-\frac{\varepsilon r}{2 r_{o}^{2}}\left(\cos \frac{2 \pi \xi}{L}-1\right)-\frac{\varepsilon g r^{3} \pi}{2 r_{o}^{2}} \sin \frac{2 \pi \xi}{L} . \quad(\xi<0)
\end{aligned}
$$

Therefore, the maximum transverse force acting on electron (moving with velocity $\approx c$ ) is given by

$$
F_{r}=-e E_{r m}+e B_{\theta m}
$$

This force causes focusing of the test electron towards the channel axis if $F_{r}<0$. The variation of the normalized transverse force $\left(F_{r} / m c \omega_{\text {po }}\right)$ with $\xi / L$ is shown in Fig. 3 for $a_{o}^{2}=0.1, \lambda_{p}=15.0 \mu \mathrm{m}, r_{o}=15.0 \mu \mathrm{m}$, and $r=$ $4.0 \mu \mathrm{m}$. Solid and dashed curves show transverse force on the electron in a plasma channel and homogeneous plasma, respectively. It may be noted that the peak trans- 


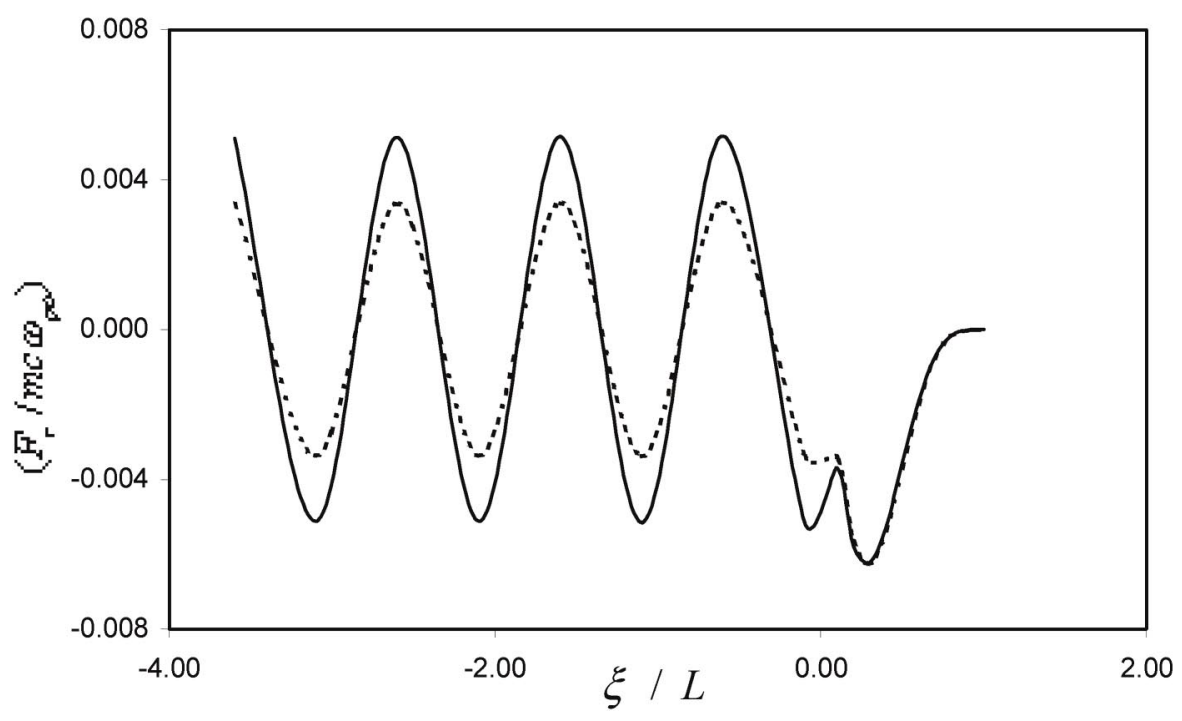

FIG. 3. Normalized transverse force $\left(F_{r} / m c \omega_{\mathrm{po}}\right)$ generated by a matched laser pulse, as a function of $\xi / L$ in plasma channel (solid curve) and in a homogeneous plasma (dashed curve) for $a_{o}^{2}=0.1, \lambda_{p}=15.0 \mu \mathrm{m}, r_{o}=15.0 \mu \mathrm{m}$, and $r=4.0 \mu \mathrm{m}$.

verse force increases (by about 52\%) in a plasma channel in comparison to homogeneous plasma due to transverse wakefields generated behind the pulse. However transverse force on a test electron within the pulse is nearly equal for both the cases.

Figure 4 shows the evolution of normalized axial and transverse forces acting on a test electron due to wakefield in a plasma channel for $a_{o}^{2}=0.1, \lambda_{p}=15.0 \mu \mathrm{m}, r_{o}=$ $15.0 \mu \mathrm{m}$, and $r=4.0 \mu \mathrm{m}$. Simultaneous acceleration and focusing will be observed in the regions where $F_{z}>0$ and $F_{r}<0$.

\section{SUMMARY AND DISCUSSION}

Wakefield generation by an intense laser pulse traveling in a parabolic plasma channel has been analyzed in detail.
This study will be significant in the development of Laser wakefield accelerators. The interaction of electric component of the laser field generates a quiver motion of the plasma electrons. Coupling of the quiver motion with the channel gradient leads to first order perturbation in the electron density. These density perturbations result in the generation of a fast oscillating axial electric field. Therefore, the transverse quiver motion of the plasma electron is superimposed by oscillation along the $z$ direction. Including this effect the equation for wakefield is set up for a radial Gaussian field amplitude and parabolic channel. For a wide channel $\left(k_{\mathrm{po}} r_{\mathrm{ch}}>1\right)$, inhomogeneity and radial derivative of transverse components of wakefields are considered as higher order effects. Electric and magnetic wakefields have been derived with the help of

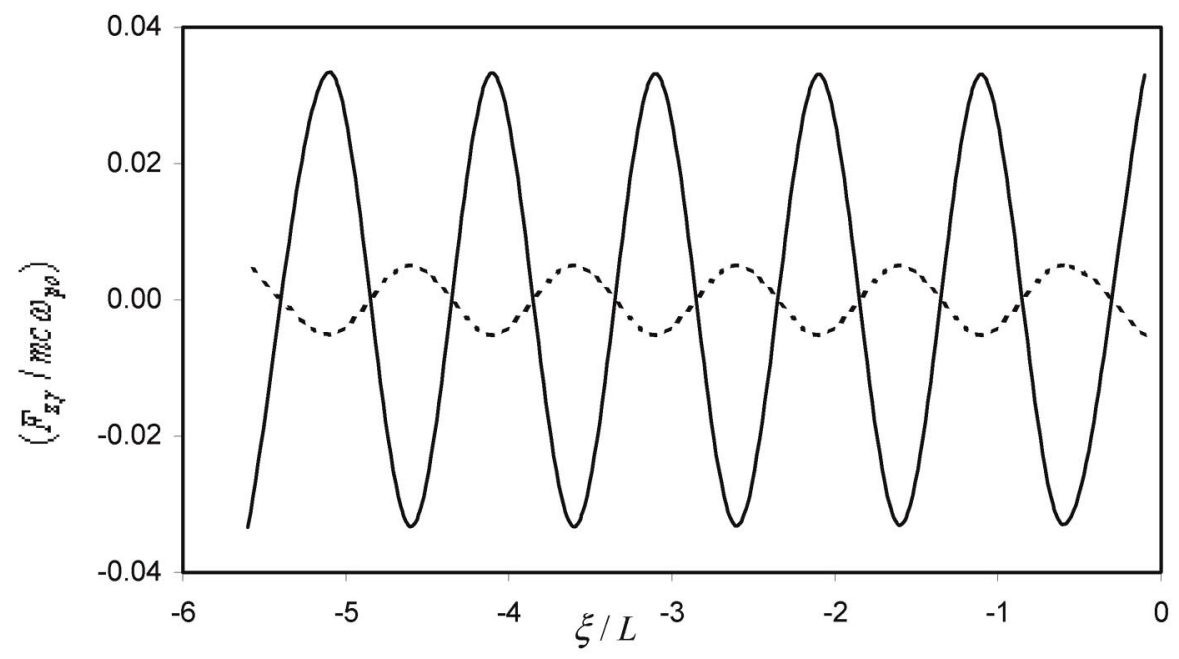

FIG. 4. Variation with $\xi / L$ of normalized axial (solid curve) and transverse (dashed curve) forces acting on a test electron behind the matched laser pulse propagating through the plasma channel for $a_{o}^{2}=0.1, \lambda_{p}=15.0 \mu \mathrm{m}, r_{o}=15.0 \mu \mathrm{m}$, and $r=4.0 \mu \mathrm{m}$. 
time dependent Maxwell's equations using quasistatic approximation. It is found that ponderomotive nonlinear effects $[\vec{\nabla}(\vec{v} . \vec{v})]$ contribute to transverse as well as longitudinal wakefield generation. However, nonlinear terms $\vec{v} \times \vec{B}$ and $\vec{v} \times(\vec{\nabla} \times \vec{v})$ representing vortex motion contribute only to transverse velocity components. Considering a sinusoidal pulse profile the zeroth and first order electric and magnetic wakefields (both transverse and longitudinal) within and behind the pulse have been obtained.

The maximum axial and transverse forces acting on a test electron due to the wakefields have been evaluated considering the pulse length to be nearly equal to plasma wavelength $\left(L \approx \lambda_{p}\right)$. The variation of these forces with $\xi / L$ have been studied graphically. For typical field and channel parameters, the maximum transverse and longitudinal forces experienced by the test electron due to wakefields generated behind the pulse are larger in a plasma channel in comparison to homogeneous plasma by $52 \%$ and $19 \%$, respectively. For wakefields generated within the pulse these forces are nearly equal. Simultaneous acceleration and focusing is observed in the regime where $F_{z}>$ 0 and $F_{r}<0$.

[1] T. Tajima and J. M. Dawson, Phys. Rev. Lett. 43, 267 (1979).

[2] P. Sprangle, E. Esarey, A. Ting, and G. Joyce, Appl. Phys. Lett. 53, 2146 (1988).

[3] P. Sprangle, E. Esarey, J. Krall, and G. Joyce, Phys. Rev. Lett. 69, 2200 (1992).

[4] E. Esarey, P. Sprangle, J. Krall, and A. Ting, IEEE Trans. Plasma Sci. 24, 252 (1996).
[5] L. M. Gorbunov, P. Mora, and A. A. Solodov, Phys. Plasmas 10, 1124 (2003).

[6] N.E. Andreev, L. M. Gorbunov, V. I. Kirsanov, and A. A. Pogosova, JETP Lett. 60, 713 (1994).

[7] D. Umstadter, J. K. Kim, and E. Dodd, Phys. Rev. Lett. 76, 2073 (1996).

[8] J. R. Marques, J. P. Geindre, F. Amiranoff, P. Audebert, J. C. Gauthier, A. Antonetti, and G.; Grillon, Phys. Rev. Lett. 76, 3566 (1996); C. W. Siders, S. P. Le Blanc, D. Fisher, T. Tajima, M. C. Downer, A. Babine, A. Stepanov, and A. Sergeev, Phys. Rev. Lett. 76, 3570 (1996); J. R. Marques et al., Phys. Rev. Lett. 78, 3463 (1997); E. Takahashi, H. Honda, E. Miura, N. Yugami, Y. Nishida, K. Katsura, and K. Kondo, Phys. Rev. E 62, 7247 (2000).

[9] F. Dorchies et al., Phys. Plasmas 6, 2903 (1999).

[10] P. Sprangle and B. Hafizi, Phys. Plasmas 6, 1683 (1999).

[11] E. Esarey, P. Sprangle, J. Krall, and A. Ting, IEEE J. Quantum Electron. 33, 1879 (1997).

[12] E. Esarey and W. P. Leemans, Phys. Rev. E 59, 1082 (1999).

[13] P. Jha, N. Wadhwani, Ajay K. Upadhyaya, and G. Raj, Phys. Plasmas 11, 3259 (2004).

[14] N.E. Andreev, L. M. Gorbunov, V.I. Kirsanov, K. Nakajima, and A. Ogata, Phys. Plasmas 4, 1145 (1997).

[15] D. C. Barnes, T. Kurki-Suonio, and T. Tajima, IEEE Trans. Plasma Sci. 15, 154 (1987).

[16] T. C. Chuiou, T. Katsouleas, C. Decker, W. B. Mori, J. S. Wurtele, G. Shvets, and J. J. Su, Phys. Plasmas 2, 310 (1995); G. Shvets, J. S. Wurtele, T. C. Chiou, and T. C. Katsouleas, IEEE Trans. Plasma Sci. 24, 351 (1996).

[17] W. Leemans, C. W. Siders, E. Esarey, N. Andreev, G. Shvets, and W. B. Mori, IEEE Trans. Plasma Sci. 24, 331 (1996).

[18] J. U. Kim, N. Hafz, and H. Suk, Phys. Rev. E 69, 026409 (2004).

[19] E. Esarey, A. Ting, P. Sprangle, and G. Joyce, Comments Plasma Phys. Control. Fusion 12, 191 (1989). 